\title{
STUDY OF EVENT MIXING AND ITS APPLICATION TO THE
}

\section{EXTRACTION OF RESONANCE SIGNALS}

D. Drijard and H.G. Fischer

CERN, Geneva, Switzerland

T. Nakada ${ }^{(*)}$

Institut für Hochenergiephysik, Heidelberg, Germany

\section{ABSTRACT}

The features of the invariant mass distribution obtained from combinations of particles from different events, i.e. event mixing, is studied in detail. It is shown that special care is needed in order to reproduce the shape of the uncorrelated invariant mass distribution. A way to determine the number of resonances and its statistical error using an event mixing technique is described.

Submitted to Nuclear Instruments \& Methods

(*) From 1 Febuary 1984 at SIN, Villigen, switzerland 


\section{INTRODTCTION}

A standard way to observe resonance production is to make an invariant mass distribution of particles which may be decay products of resonances. If resonances are produced, a peak will appear in the distribution on top of a background which comes from combinations of nonresonating particles. In order to determine the number of resonances from such a distribution, an estimation of the background is essential. If the background has a simple falling or rising form, it will not be difficult to approximate the shape of the background below the resonance signal with an analytic function.

However, some resonances sit just at the place where the whole invariant mass distribution peaks. In this case it is very difficult to estimate the exact shape of the background and it was shown that different choices of functions which approximate the shape of the background may change the estimation of the resonance content as much as $60 \%$ [1].

Furthermore in the case of experiments with electronics detectors, un1ike in the case of the bubble chamber experiments, there is always an acceptance problem of the detector. This may create an artificial structure in the invariant mass distribution.

In order to overcome these problems, the mass distribution with combinations of particles from different events, "event mixing", is used to estimate the background [2]. However, there is an indication that a simple subtraction of the mass distribution obtained with event mixing cannot give the final answer [3].

In this paper we study in detail the features of the mass distribution from event mixing and show how to use it for an estimation of the background. A statistical model is used to understand the statistical behaviour of the mass distribution from event mixing which is rather complicated due to a possible correlation with original events. Finally, a method to determine the number of resonances and its statistical error using an event mixing technique is described. An example for the application of this method can be found elsewhere [4]. 


\section{KINEMATICS OF EVENT MIXING}

\subsection{General concept}

Suppose an object $A$ with a mass $M$ decaying into two particles $B$ and $C$. $A$ is produced at rest and only those events where $B$ is emitted to a fixed angle are accepted. Then the invariant mass of the system $B-C$ is also equal to $M$ for $B$ and $C$ coming from different events, i.e. event mixing (fig. 1).

Nów suppose $A$ is produced still at rest but all events are accepted, i.e. B may go into any direction. Then the invariant mass of the system B-C for event mixing is no longer always equal to $M$ (fig. 2). The distribution of those invariant masses is broad and is maximum at $M$.

When a moves toward a fixed direction, say toward the Z-axis, the invariant mass distribution of the system $B-C$ from event mixing becomes broader than in the preceding case and the maximum is beyond $M$. In general the direction of $A$ is not fixed, then the mass distribution from event mixing becomes even broader.

The above observation can be generalized in the following way: increasing the freedom in kinematics for the secondary particles introduces an increasing distortion in the invariant mass distribution from event mixing, as compared with the original mass distribution. The details of this broadening effect depend on the shape of the original invariant mass distribution. The broader is the original mass distribution, the less sensitive it is to the distortion introduced by event mixing.

Therefore, in the case of a broad background invariant mass distribution, event mixing may reproduce the original mass distribution if the kinematical range of secondaries is sufficiently restricted. However, a resonance, whose mass distribution is narrower than that of the background, will be distorted by event mixing if we apply the same kinematical restrictions (fig. 3 ).

Now the distribution obtained with event mixing is subtracted from the original distribution (both normalized to the same area). In the case 
of background, only a statistical fluctuation around 0 is expected for the distribution of the difference. For the resonances, the following structure is expected. At around the maximum of the original distribution a peak above 0 appears after the subtraction. At both tails, since the subtracted distribution is too wide, as it was explained, dips will be created. This becomes clear from fig. 4. The solid line indicates the resonance and the solid line with crosses comes from event mixing. A is the excess area of the original distribution over that from event mixing and $A$ ' is the overlapping area. $B$ and $C$ are the areas where the distribution from event mixing exceeds the original one. Since the two distributions are equally normalized, $A=B+C$. After the subtraction those areas are transformed into the second figure.

The relative importance of the area $A$ depends on the shape of the invariant mass distribution obtained with event mixing. Therefore, it depends on the kinematical restrictions applied, e.g. in $P_{t}$, $\Phi$ and Feynman- $x$, and the shape of the differential cross section of the resonance. For example, if the $P_{t}$ distribution of the resonance is broader, there will be more kinematical freedom and invariant mass distribution from event mixing will become wider, i.e. A will increase.

\subsection{Simulation study}

These qualitative arguments can be demonstrated using a simulation method. Let us take $\Delta^{++}$production in $p-p$ interactions as an example. $\Delta^{++}$events are generated according to a p-wave Breit-wigner function [5] and $\mathrm{d}^{2} \sigma / d x d P_{t}^{2} \propto \exp \left(-4 P_{t}\right)$ in one $x$ hemisphere. This parametrization is suggested by the measurements of various baryon production at ISR [6-8]. All $\Delta^{++}$generated decays into $p$ and $\pi^{+}$. Background events are generated such that the measured inclusive $p$ and $\pi^{+}$cross sections in $p-p$ interactions at ISR $[6,8]$ are approximately satisfied. Since we are interested in the inclusive process, the energy momentum conservation is not taken into account, but the total $\mathrm{p}^{+}$energy is kept below half the total energy $\mathrm{Vs}$.

Fig. S(a) shows the invariant mass distribution of these "background" $\mathrm{p} \pi^{+}$systems. No kinematical constraint was applied. Fig. $5(\mathrm{~b})$ shows the $\mathrm{pr}^{+}$mass distribution obtained with mixing the background events. 
In the mixing, on1y those $\mathrm{pr}^{+}$pairs whose energies are below $\mathrm{vs} / 2$ are taken. Fig. $5(c)$ shows the mass spectrum after the subtraction of fig. $5(b)$ from fig. 5(a). As one can clearly see, a structure emerges after the subtraction due to the broadening effect of the mass spectrum from event mixing. Fig. 6 is also the $\mathrm{pr}^{+}$mass distribution after the subtraction of that obtained by event mixing, with the following kinematical cuts in $x$ for secondaries applied for both the original and mixed events: $0.3<\mathrm{x}(\mathrm{P})<0.4$ and $0.0<\mathrm{x}\left(\pi^{+}\right)<0.1$. Now there is no systematic structure. This shows that the constraints applied are tight enough to reproduce the shape of the original mass distribution with event mixing.

How does this procedure work for the resonance? Fig. $7(a)$ is the $p \pi^{+}$ mass spectrum for decays of generated $\Delta^{++}$, where $0.3<x(p)<0.4$ and $0.0<x\left(\pi^{+}\right)<0.1$, and similarly fig. $7(b)$ for event mixing. The broadening of the mass distribution in the case of event mixing is clearly seen. After the subtraction, fig. $7(\mathrm{c})$ is obtained where we can see the characteristic structure described previously (fig. 4).

It becomes clear that the cuts which are tight enough to reproduce the background, stil1 distort the shape of the mass spectrum in the case of the resonance.

In an experiment there are both resonance and background and the correlation between them introduced by event mixing must be considered. Fig. 8 shows the $\mathrm{pr}^{+}$invariant mass distribution where the same number of $\Delta^{++}$were generated as in the case of fig. 7 , together with background in the ratio (resonance/background) $=1 / 9$. After the subtraction of the mass distribution from event mixing the second figure is obtained. The structure is similar to that of $\mathrm{fig} .7$ where there are only $\Delta^{++}$. The numbers of entries above 0 in both figures are equal within statistics. Therefore, we conclude that the resonance and the background are properly separated in event mixing subtraction.

\section{STATISTICAL BEHAVIOUR OF EVENT MIXING}

Let us study this problem of the event mixing quantitatively, using an argument based on statistics. We define $n_{0}$ and $n_{e m}$ to be the total 
number of $\mathrm{pr}^{+}$combinations for the original and mixed events, respectively. Introducing a restriction on the mass range, $(m, m+\Delta m)$, we define similarly $n_{0}(m)$ and $n_{e m}(m)$ within this mass range. In order to gain statistical precision, we combine in event mixing the $\pi^{+}$from one event with the protons from $k$ other events, so that $n_{0}=n_{e m} / k$. We introduce further

$$
n_{s}(m)=n_{0}(m)-n_{e m}(m) / k
$$

The distribution of the random variable $n_{s}(m)$ carries the relevant information to estimate the resonance signal and we are going to study this distribution in terms of expectation value $E$ and variance $V$.

One can show that the expectation values of $n_{0}(m), n_{e m}(m)$ and $n_{s}(m)$ can be expressed in terms of the following eleven probabilities.

For the original events,

a the probability to have $\Delta^{++}$resonance,

$p$ the probability for the $\Delta^{++}$mass to be between $m$ and $m+\Delta m$,

$q$ the probability for the mass of a background $\mathrm{pr}^{+}$ combination to be between $m$ and $m+\Delta m$,

and for mixed events, the probabilities that the $\mathrm{pr}^{+}$masses of the following mixings between two events are between $m$ and $m+\Delta m$.

\section{Event 1}

$$
\begin{array}{ll}
\mathrm{a} & \mathrm{p}\left(\Delta^{++}\right) \\
\mathrm{b} & \mathrm{p}\left(\operatorname{non} \Delta^{++}\right) \\
\mathrm{c} & \mathrm{p}\left(\Delta^{++}\right) \\
\mathrm{d} & \mathrm{p}\left(\operatorname{non} \Delta^{++}\right) \\
\mathrm{e} & \mathrm{p}\left(\Delta^{++}\right) \\
\mathrm{f} & \mathrm{p}\left(\operatorname{non} \Delta^{++}\right) \\
\mathrm{g} & \mathrm{p}\left(\Delta^{++}\right) \\
\mathrm{h} & \mathrm{p}\left(\operatorname{non} \Delta^{++}\right)
\end{array}
$$

\section{Event 2}

$$
\begin{aligned}
& \pi^{+}\left(\Delta^{++}, \text {inside }\right) \\
& \pi^{+}\left(\Delta^{++}, \text {inside }\right) \\
& \pi^{+}\left(\Delta^{++}, \text {outside }\right) \\
& \pi^{+}\left(\Delta^{++}, \text {outside }\right) \\
& \pi^{+}\left(\text {non } \Delta^{++}, \text {inside }\right) \\
& \pi^{+}\left(\text {non } \Delta^{++}, \text {inside }\right) \\
& \pi^{+}\left(\text {non } \Delta^{++}, \text {outside }\right) \\
& \left.\pi^{+} \text {(non } \Delta^{++}, \text {outside }\right)
\end{aligned}
$$


where " $\Delta^{++} "$ means that the particle is from a decay of $\Delta^{++}$and "inside" means that the $\pi^{+}$is from a $\mathrm{p \pi}^{+}$pair with invariant mass between $m$ and $m+\Delta m$ in the original events. The results are the following:

$$
\begin{aligned}
& E\left[n_{o}(m)\right]=\mu\{\alpha p+(1-\alpha) q\}, \\
& E\left[n_{e m}(m)\right]=\mu k\{\alpha p w+(1-\alpha) q y+\alpha(1-p) x+(1-\alpha)(1-q) z\}, \\
& E\left[n_{s}(m)\right]=E\left[n_{0}(m)\right]-E\left[n_{e m}(m)\right] / k,
\end{aligned}
$$

where $E[X]$ is the expectation value of $X$ and

$$
\begin{aligned}
& w=a \alpha+b(1-\alpha), \\
& x=c \alpha+d(1-\alpha), \\
& y=e \alpha+f(1-\alpha), \\
& z=g \alpha+h(1-\alpha) .
\end{aligned}
$$

The parameter $\mu$ represents the mean of the Poisson law followed by $n_{0}$ : $E\left[n_{0}\right]=V\left[n_{0}\right]=\mu$. Details of the calculation can be found elsewhere $[9]$. The variances of $n_{0}(m), n_{e m}(m)$ and $n_{s}(m)$ can be also expressed as

$$
\begin{aligned}
& V\left[n_{0}(m)\right]=\mu\{\alpha p+(1-\alpha) q\} \text {. } \\
& V\left[n_{e m}(m)\right]=\mu k\{\alpha p w\{1+w(k-1)\}+(1-\alpha) \text { qy }\{1+y(k-1)\} \\
& +\alpha(1-p) \times\{1+x(k-1)\}+(1-\alpha)(1-q) z\{1+z(k-1)\}] \text {, } \\
& V\left[n_{s}(m)\right]=\mu\{\alpha p+(1-\alpha) q\}+\mu[\alpha p w\{w+(1-w) / k\} \\
& +(1-\alpha) \text { qy }\{y+(1-y) / k\}+\alpha(1-p) x\{x+(1-x) / k\} \\
& +(1-\alpha)(1-q) z\{z+(1-z) / k\} 1-2 \mu\{\alpha p w+(1-\alpha) q y\} \text {. }
\end{aligned}
$$

Let us now concentrate on $E\left[n_{s}(m)\right]$ and $V\left[n_{s}(m)\right]$. Among the eleven probabilities which we defined previously, $\alpha$ is what we want to determine from the data and $p$ can be calculated with a simulation, since it is related on1y to the resonance. Now we are left with nine probabilities.

We here assume that $p$ and $\pi^{+}$, one from $a \Delta^{++}$and the other from the background in the mixed events, are equally uncorrelated as $p$ and $\pi^{+}$from the background in the original events. Then the nine probabilities become a11 equal to the probability $t$, which is the probability that any uncorrelated $\mathrm{pr}^{+}$pair has a mass between $\mathrm{m}$ and $\mathrm{m}+\Delta \mathrm{m}$. Then we have

$$
\mathrm{t}=\mathrm{a}=\mathrm{b}=\mathrm{c}=\mathrm{d}=\mathrm{e}=\mathrm{f}=\mathrm{g}=\mathrm{h}=\mathrm{q} \text {. }
$$


Since we have just assumed that in event mixing everything is uncorrelated, $t$ can be calculated also from a simulation, doing event mixing only with $\Delta^{++}$.

Then $E\left[n_{0}(m)\right], E\left[n_{e m}(m)\right], E\left[n_{s}(m)\right]$ and $V\left[n_{s}(m)\right]$ become

$$
\begin{aligned}
& E\left[n_{o}(m)\right]=\{\alpha p+(1-\alpha) t\} \mu, \\
& E\left[n_{e m}(m)\right]=\mu k t, \\
& E\left[n_{s}(m)\right]=\mu \alpha(p-t), \\
& V\left[n_{s}(m)\right]=\mu\{\alpha p+(1-\alpha) t\}(1-2 t)+\{t+(1-t) / k\} \mu t,
\end{aligned}
$$

and the variance can be expressed with the expectation values as

$$
\begin{aligned}
V\left[n_{s}(m)\right]= & \left\{E^{2}\left[n_{e m}(m)\right] E\left[n_{0}-n_{0}(m)\right]+E^{2}\left[n_{e m}-n_{e m}(m)\right]\right. \\
& E\left[n_{0}(m)\right]+E^{2}\left[n_{0}\right] E\left[n_{e m}(m)\right] E\left[n_{e m}-n_{e m}(m)\right] / \\
& \left.E\left[n_{e m}\right]\right\} / E^{2}\left[n_{e m}\right] .
\end{aligned}
$$

For the estimation one can replace the expectation values with the experimentally observed values and we obtain

$$
\begin{aligned}
N_{s}=\mu \alpha(p-t) & \\
V\left[n_{s}(m)\right] & =\left[N_{e m}^{2}(m)\left\{N_{0}-N_{0}(m)\right\}+\left\{N_{e m}-N_{e m}(m)\right\}^{2} N_{0}(m)\right. \\
& \left.+N_{0}^{2} N_{e m}(m)\left\{N_{e m}-N_{e m}(m)\right\} / N_{e m}\right] / N_{e m}^{2},
\end{aligned}
$$

where the capital $\mathrm{N}$ means the observed value. Now the variance of $n_{s}(\mathrm{~m})$ is expressed only with observed numbers.

\section{EXTRACTION OF THE SIGNAL}

One can of course use eq. (3.2) at a certain mass $m$ to determine the number of resonances $\alpha \mu$, for instance the region where the entries are above 0 in fig. 8. However, in this way on $1 y$ a part of the information is used and one gains more in precision, when using the total mass spectrum of fig. 8 . 
We go back to the simulation results done in sect. 2.2. The aim is to extract the number of resonances from fig. 8 , where both $\Delta^{\text {t+ }}$ and background are generated. After all the previous arguments, it is now clear that the structure in fig. 8 is only due to $\Delta^{++}$because of the appropriate kinematical cuts for the secondaries. Then we can fit the mass distribution in fig. 8 to that in fig. 7 , and $x^{2}$ is given by

$$
x^{2}=\sum_{m}\left[\left\{N_{s}(m)-n_{\Delta}++N_{m c s}(m) / N_{\Delta}++\right\}^{2} / \delta_{m}^{2}\right],
$$

where $N_{s}(m)$ and $N_{m c s}(m)$ are the number of entries in the p* mass distribution between $m$ and $m+\Delta m$ after the subtraction of event mixing for the data, fig. 8 in this case, and for the reference fig. 7 respectively. $N_{\Delta}++$ is the number of $\Delta^{++}$. generated in the reference sample and $n_{\Delta}++$ is the free parameter which gives the number of $\Delta^{++}$in fig. 8. If the reference distribution fig. 7 is generated with high statistics, its contribution to the statistical error $\delta_{m}$ is negligible. Therefore,

$$
\delta_{\mathrm{m}}=\sqrt{\mathrm{v}\left[\mathrm{n}_{\mathrm{s}}(\mathrm{m})\right]},
$$

where $V\left[n_{s}(m)\right]$ is given by eq. (3.3).

Solving

$$
\partial x^{2} / \partial n_{\Delta}++=0
$$

we get

$$
n_{\Delta}++=N_{\Delta}++\left[\sum_{m}\left\{N_{s}(m) N_{m c s}(m) / \delta_{m}^{2}\right\} /\left\{\sum_{m}, N_{m c s}^{2}\left(m^{*}\right) / \delta_{m^{\prime}}^{2}\right\}\right],
$$

and for $\sigma$, the error on $n_{\Delta}++$, neglecting the correlation between $N_{s}(m)$ and $N_{s}\left(m^{\prime}\right)$, where $m \neq m^{\prime}$ and taking the number of mass bins large enough, one gets

$$
\sigma^{2}=\sum_{m}\left[\left\{\partial n_{\Delta}++\partial N_{s}(m)\right\}^{2} \delta N_{S}^{2}(m)\right] .
$$

As we have seen $\delta N_{S}(m)=\delta_{m}$ and finally we derive

$$
\sigma^{2}=N_{\Delta}^{2}++\left[1 / \sum_{m}\left\{N_{m c s}^{2}(m) / \delta_{m}^{2}\right\}\right] \text {. }
$$

\section{TEST OF THE FITTING METHOD WITH SIMULATION}

Finally, we check our results in detail with the simulation described in sect. 2.2. For this study 100 different samples are generated for each of the following three different event types: 
(a) $300 \Delta^{++}$events,

(b) 3000 background events,

(c) $300 \mathrm{\Delta}^{++}$and 3400 background events.

$\mathrm{pr}^{+}$mass distributions are made with $20 \mathrm{MeV} / \mathrm{c}^{2}$ bin with both for the original and mixed events. The reference distribution is made with $10000 \Delta^{++}$events. The kinematical cuts which are applied for secondaries are $0<\mathrm{x}\left(\pi^{+}\right)<0.1$ and $0.4<\mathrm{x}(\mathrm{p})<0.5$.

The simulation study shows that our assumption for the nine probabi1ities, i.e. eq. (3.1), holds. The error formula eq. (3.3) is then checked. The r.m.s. of the distribution of entries in a certain mass bin of the $\mathrm{p}^{+}$mass distribution over 100 samples, where the mass spectrum obtained from event mixing is subtracted from that of the original events, is compared to the average of the errors calculated with eq. (3.3). They agree with each other within statistics for all three different event types. These results are also true with different kinematical restrictions for $p$ and $\pi^{+}$.

From this study we conclude that eq. (3.3) gives us a reasonable statistical error for the entries of the $\mathrm{pr}^{+}$mass distribution, where the mass distribution from event mixing is subtracted from the original mass distribution.

Finally, we test how well we can determine the number of $\Delta^{++}$with eq. (4.1) and its error with eq. (4.2). Table 1 shows the results of the test. Here, $\overline{n_{\Delta^{++}}}$is the number of $\Delta^{++}$averaged over 100 samples obtained with eq. (4.1) and $\bar{\sigma}$ is calculated with eq. (4.2) and averaged over 100 samples. Then the r.m.s., $\hat{o}$, is determined from the distribution of $n_{\Delta^{++}}$around $\overline{n_{\Delta}^{++}}$over the 100 samples.

Table 1 shows a very good agreement between $\overline{n_{\Delta^{++}}}$and the generated number of $\Delta^{++}$(since we averaged over 100 samples, the uncertainty on $\overline{n_{\Delta^{++}}}$ is $\bar{\sigma}(10)$. Also $\bar{\sigma}$ agrees very well with $\hat{o}$. As one notices, for the event type 1 and the event type 3 , which contain the same number of $\Delta^{++}$, eq. (4.1) gives the same result which again proves the absence of the correlation between $\Delta^{++}$and background in event mixing. 
When one applies this fitting procedure to the experimental data, the systematic error must be considered in addition to the statistical error given by eq. (4.2). Possible contribution to the systematic error may come from the detector, e.g. a limited accuracy of the momentum measurement and misaligment of the detector components. The assumed parametrization for the invariant cross section of the resonance in the simulation, used to produce the reference mass distribution which is fitted to the data, may also introduce a further systematic error. Those effects can be estimated by the simulation and such a study can be found elsewhere $[9]$.

\section{CONCLUSIONS}

In this paper we have shown how event mixing can be used in order to extract a resonance signal. Although $p \pi^{+}$mass spectra and $\Delta^{++}$resonances are used as an example, all the arguments and results are valid in any mass spectrum and resonance.

It was commonly believed that event mixing reproduced an uncorrelated background mass distribution. However, our study shows that appropriate kinematical constraints must be applied to the secondaries in order to reproduce the background in event mixing. However, the restrictions must be not too tight for the extraction of a resonance signal. Otherwise, the shape of the resonance mass distribution is also reproduced with event mixing and we are not able to observe the signal.

Once the appropriate kinematical constraints are imposed, the difference between the original mass distribution and the one obtained with event mixing is only due to the resonance. Thus, although event mixing does not provide the background, the difference from the background can be taken into account by the simulation study of the resonance. 


\section{REFERENCES}

[1] P.D. Higgins et a1., Phys. Rev. D19 (1979) 731.

[2] See for example G. Jancso et a1., Nuc1. Phys. B124 (1977) 1.

[3] P.D. Higgins et a1., Phys. Rev. D19 (1979) 65.

[4] A. Breakstone et al., CERN/EP 83-5 Rev. to be published in Zeitschr. für Phys. C.

[5] J.D. Jackson, Nuovo Cimento 34 (1964) 1644.

[6] M.G. Albrow et a1., Nuc1. Phys. B73 (1974) 40.

[7] J. Engler et a1., Nuc1. Phys. B84 (1975) 70;

S. Erhan et a1., Phys. Lett. B85 (1979) 447;

D. Drijard et al., Zeitschr. für Phys. C12 (1982) 217;

A.E. Brenner et al., Phys. Rev. D26 (1982) 1497;

G.J. Bobbink et al., Nuc1. Phys. B217 (1983) 11.

[8] B. Alper et al., Nuc1. Phys. B100 (1975) 237.

[9] T. Nakada, CERN/EP/Internal Report 83-10 (1983). 
TABLE 1

Numbers of $\Delta^{++}$and errors

\begin{tabular}{|c|c|c|c|c|}
\hline Event type & $\overline{n_{\Delta}++}$ & Generated $\Delta^{++}$ & $\bar{\sigma}$ & $\hat{\sigma}$ \\
\hline 1 & 305.0 & 300 & 27 & 26 \\
2 & 3.6 & 0 & 62 & 64 \\
3 & 305.0 & 300 & 75 & 78 \\
\hline
\end{tabular}




\section{EIGURE CAPTIONS}

Fig. 1 Two original events and one mixed event where the particle $A$ at rest decays into two particles $B$ and $C$. $B$ and $C$ are emitted always to the same direction.

Fig. 2 Same as fig. 1 but $B$ and $C$ go into any direction.

Fig. 3 Expected mass distribution of original and mixed events for background and resonances.

Fig. 4 Expected mass distribution of resonances for original and mixed events. The second figure is obtained by the subtraction of the mixed events mass distribution from the original one.

Fig. 5 Simulated $\mathrm{p \pi}^{+}$invariant mass distribution of background for: (a) original, (b) mixed events and (c) after the subtraction of that from event mixing from the original distribution with equal normalizations. No cut is applied and $\sigma$ is calculated from eq. (3.3).

Fig. 6 Same as fig. $S(c)$ but with the following kinematical cuts for both original and mixed events: $0.3<x(p)<0.4$ and $0.0<x\left(\pi^{+}\right)<0.1$. Again $\sigma$ is calculated from eq. (3.3).

Fig. 7 simulated $\mathrm{pr}^{+}$invariant mass distribution of decays from $\Delta^{++}$ resonances for: (a) original events, (b) mixed events and (c) after the subtraction of the second figure from the first one, where the second figure is normalized to the first one.

Fig. 8 Same as fig. $7(\mathrm{c})$ but both background and $\Delta^{++}$are simulated together. The number of generated $\Delta^{++}$is the same as in $f i g .7$. 


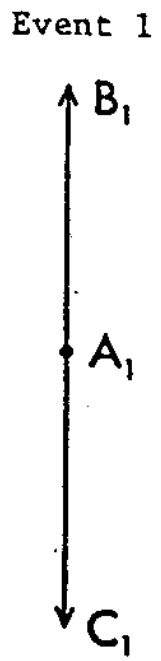

Event 2

Mixed event
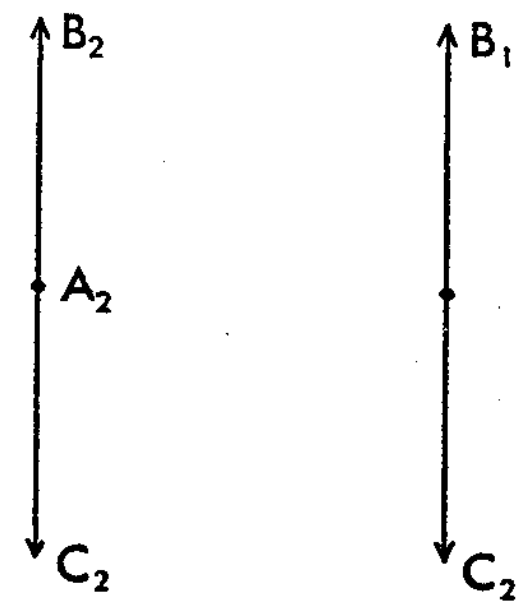

Fig. I

Event 1

Event 2

Mixed event
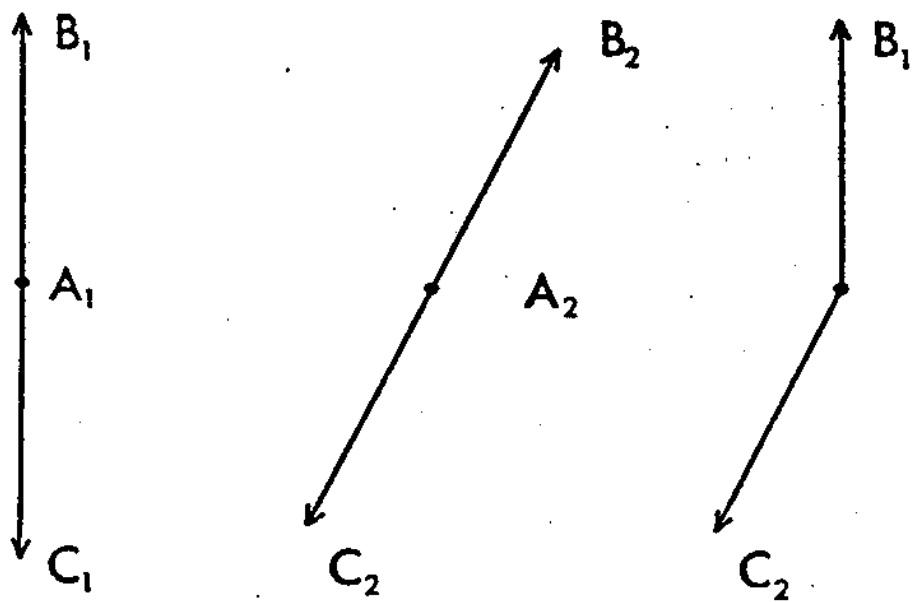

Fig. 2 

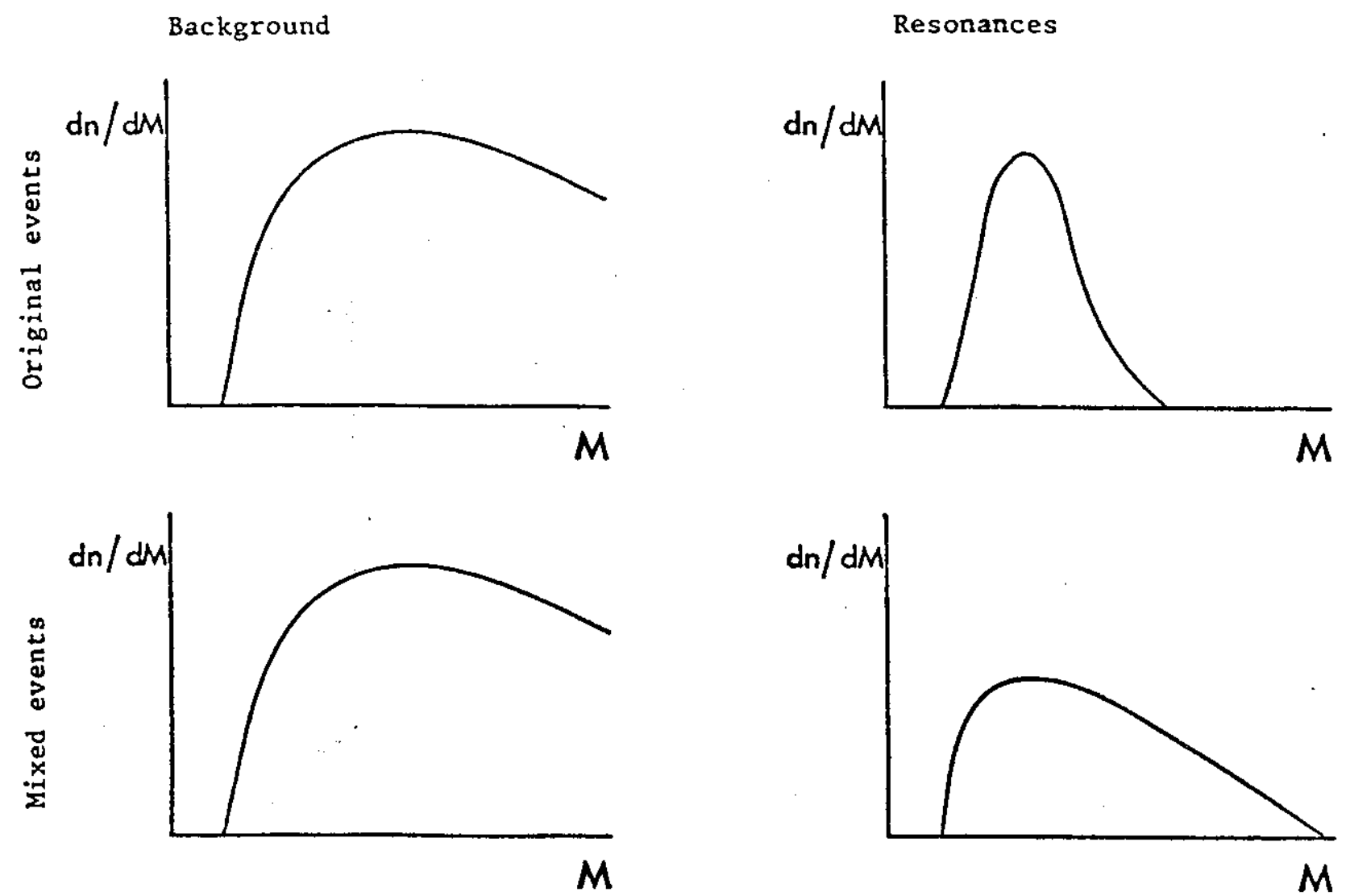

Fig. 3
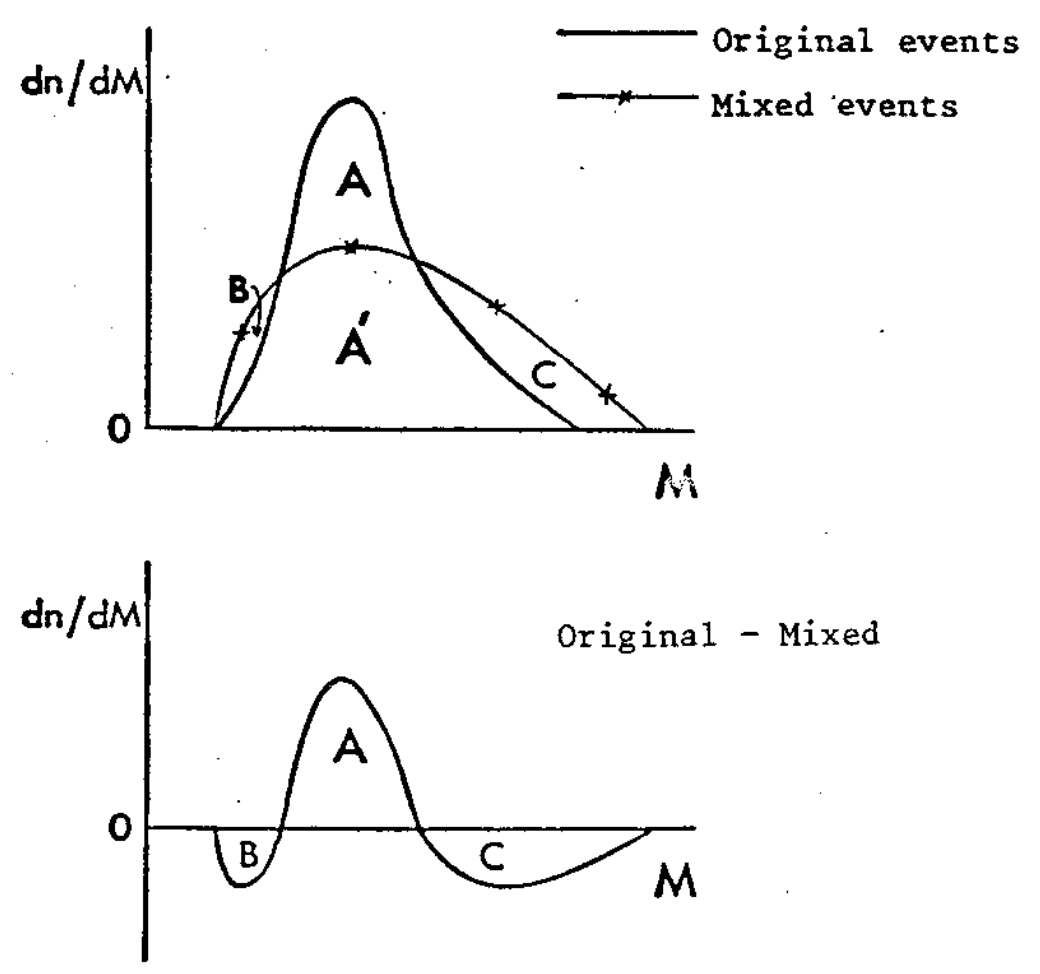

Fig. 4 


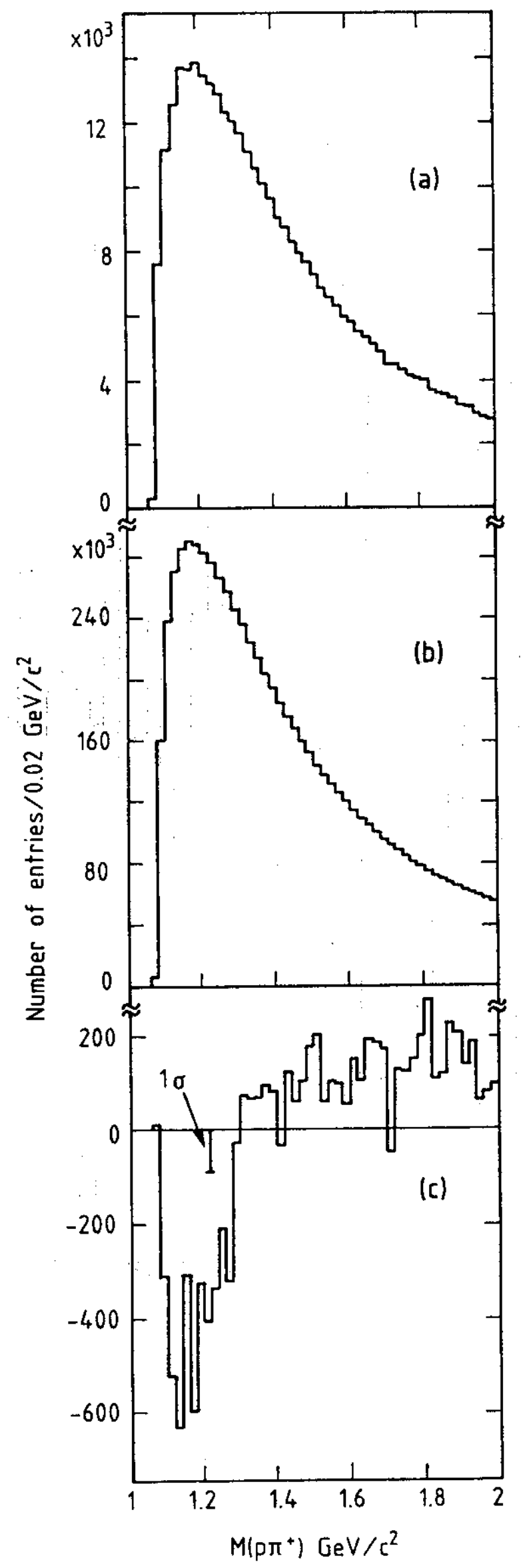

Fig. 5 


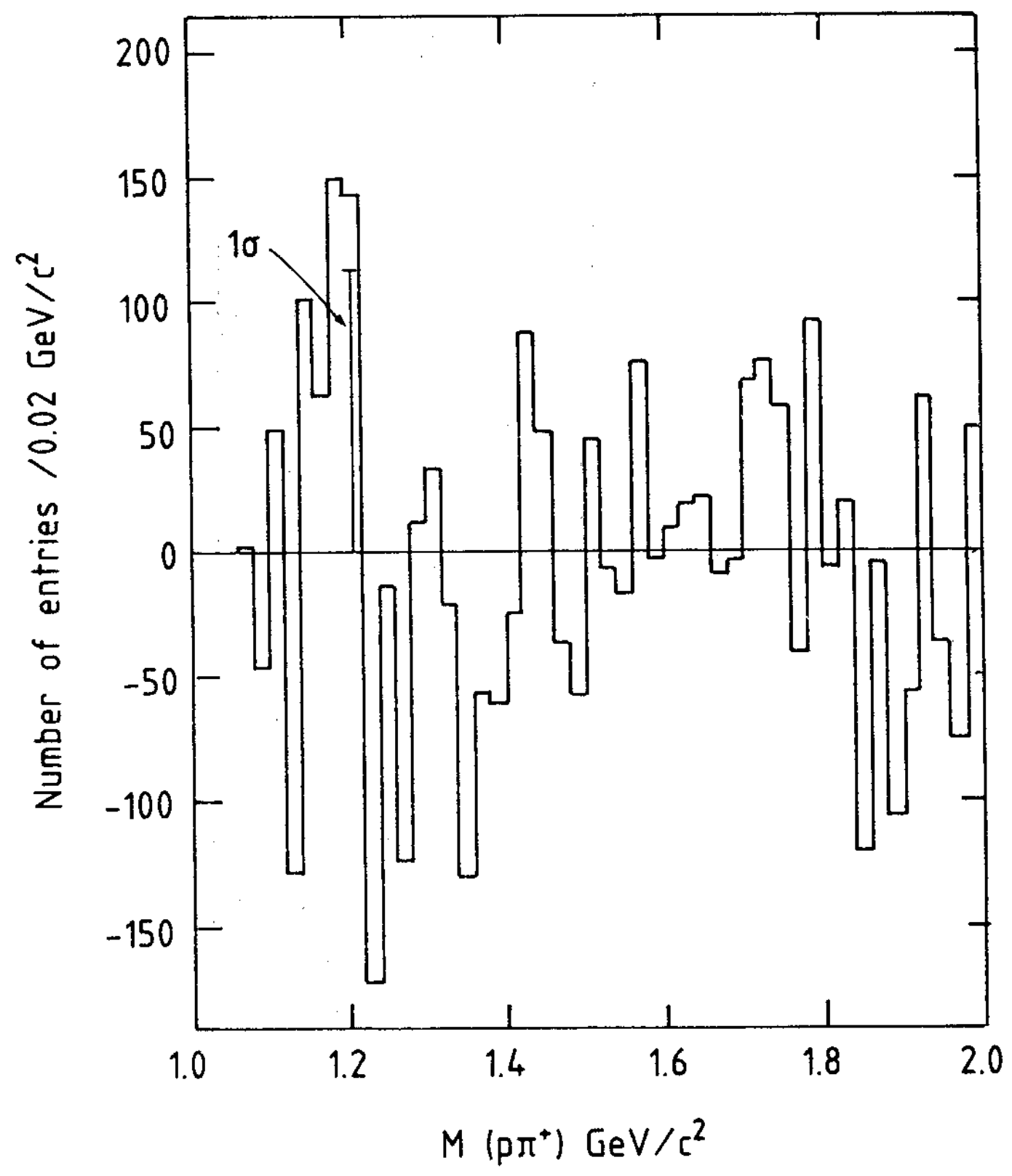

Fig. 6 


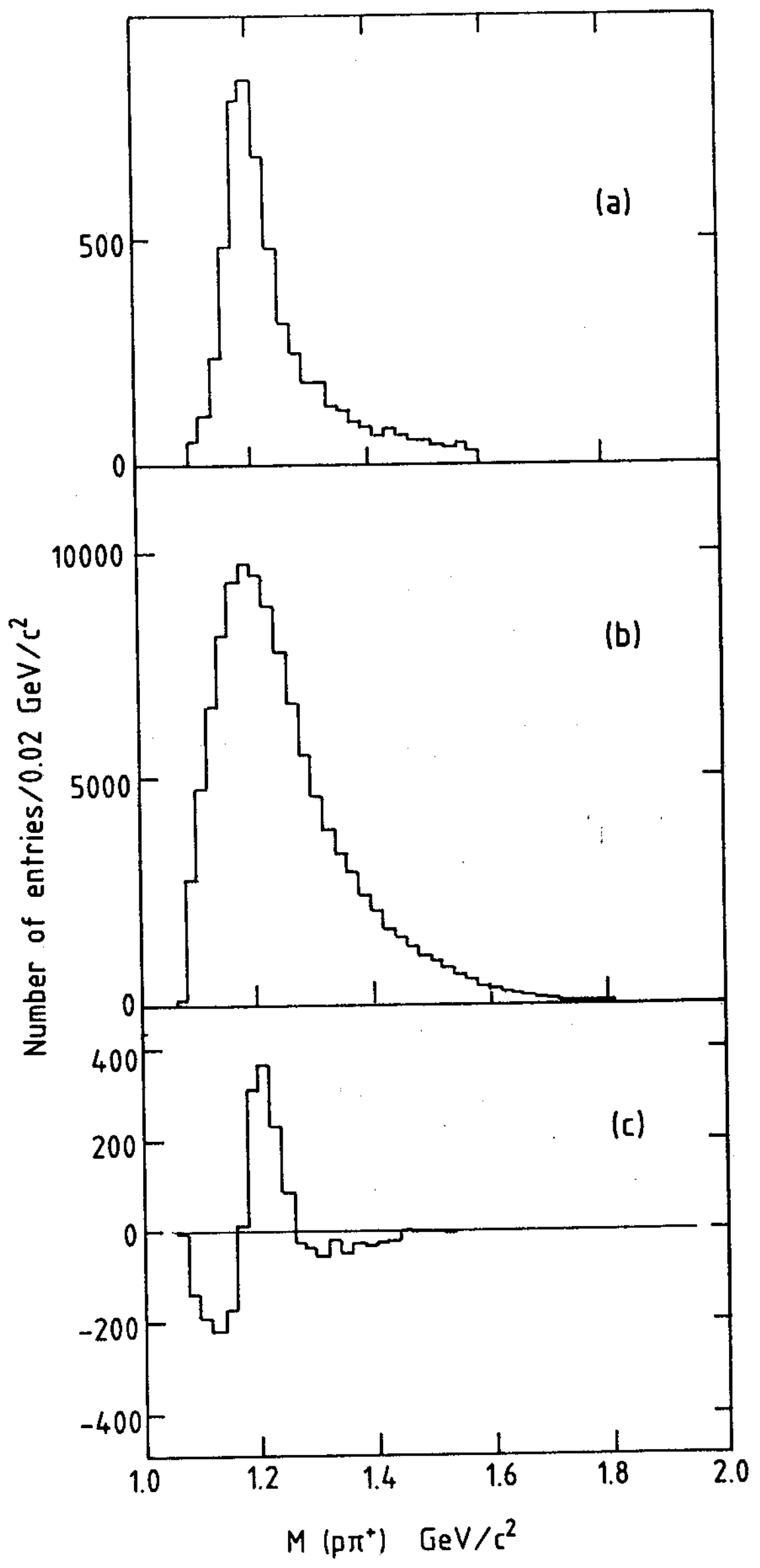

Fig. 7 


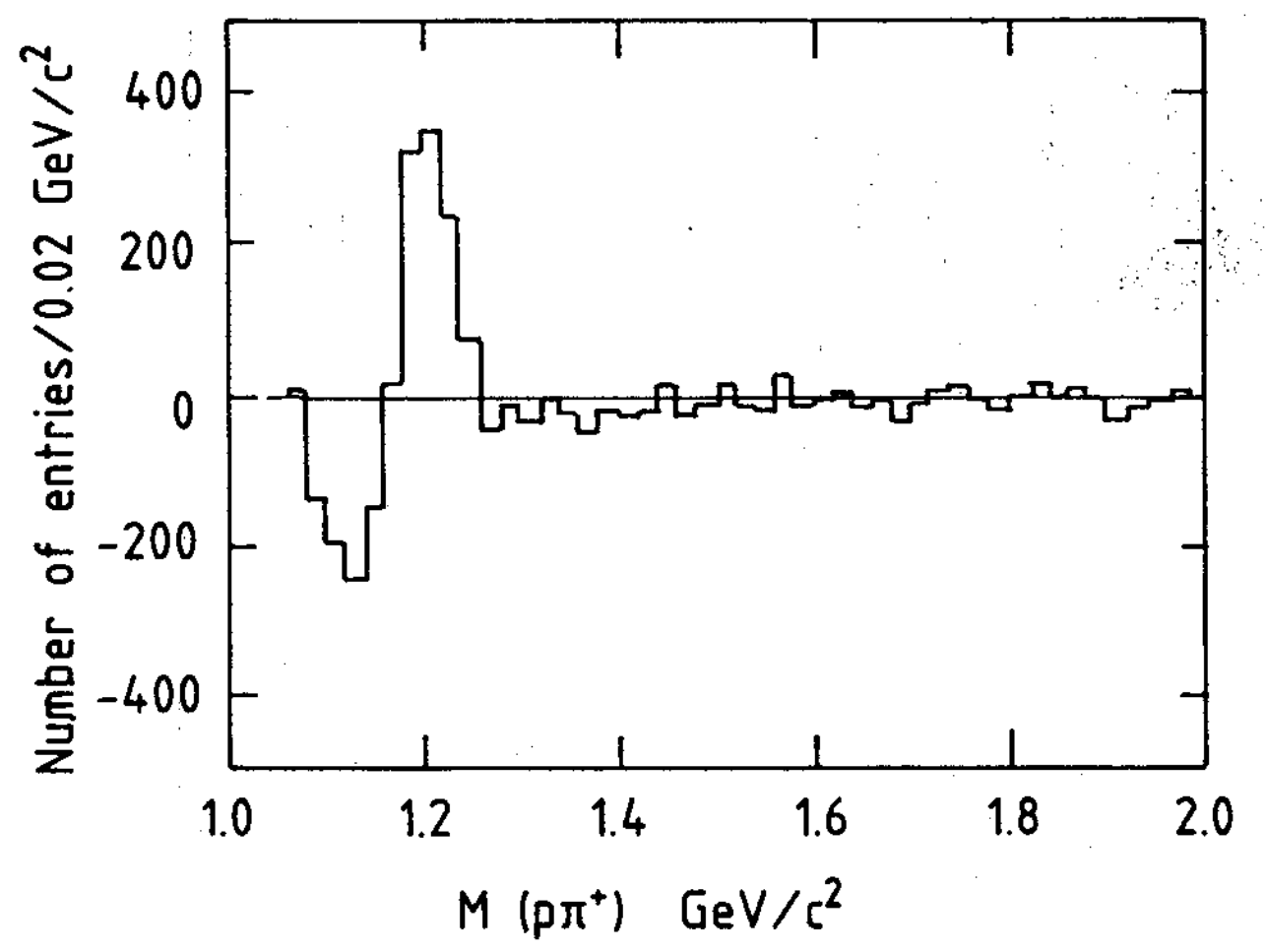

Fig. 8 